\title{
Erratum to: The role of theory building in the teaching of secondary geometry
}

\author{
Michael Weiss $^{1} \cdot$ Patricio Herbst $^{2}$
}

Published online: 1 April 2015

(C) Springer Science+Business Media Dordrecht 2015

Erratum to: Educ Stud Math (2015)

DOI 10.1007/s10649-015-9599-x

The original version of this article contained an error in the presentation of Theorem 2 (Ceva's Theorem). The corrected presentation is given below.

Theorem 2 (Ceva's Theorem) Consider triangle $A B C$ with points $P$ on $\overleftrightarrow{A B}, Q$ on $\overleftrightarrow{B C}$, and $R$ on $\overleftrightarrow{C A}$. Then lines $\overleftrightarrow{A Q}, \overleftrightarrow{B R}$, and $\overleftrightarrow{C P}$ are concurrent if and only if

$$
\frac{\langle A P\rangle}{\langle P B\rangle} \quad \frac{\langle B Q\rangle}{\langle Q C\rangle} \quad \frac{\langle C R\rangle}{\langle R A\rangle}=1
$$

The online version of the original article can be found at http://dx.doi.org/10.1007/s10649-015-9599-x.

Patricio Herbst

pgherbst@umich.edu

Michael Weiss

mweiss@msu.edu

1 College of Education, Michigan State University, East Lansing, MI, USA

2 School of Education, University of Michigan, Ann Arbor, MI, USA 